\title{
Co-synergism of endophyte Penicillium resedanum LK6 with salicylic acid helped Capsicum annuum in biomass recovery and osmotic stress mitigation
}

Abdul Latif Khan ${ }^{1,2,3}$, Muhammad Waqas ${ }^{2}$, Muhammad Hamayun ${ }^{4}$, Ahmed Al-Harrasi ${ }^{1}$, Ahmed Al-Rawahi ${ }^{1}$ and In-Jung Lee 2* $^{*}$

\begin{abstract}
Background: Water-deficiency adversely affects crop growth by generating reactive oxygen species (ROS) at cellular level. To mitigate such stressful events, it was aimed to investigate the co-synergism of exogenous salicylic acid (SA) and symbiosis of endophytic fungus with Capsicum annuum L. (pepper).

Results: The findings of the study showed that exogenous SA $\left(10^{-6} \mathrm{M}\right)$ application to endophyte (Penicillium resedanum $L K 6$ ) infected plants not only increased the shoot length and chlorophyll content but also improved the biomass recovery of pepper plants under polyethylene glycol (15\%) induced osmotic stress (2, 4 and 8 days). Endophyte-infected plants had low cellular injury and high photosynthesis rate. SA also enhanced the colonization rate of endophyte in the host-plant roots. Endophyte and SA, in combination, reduced the production of ROS by increasing the total polyphenol, reduce glutathione, catalase, peroxidase and polyphenol oxidase as compared to control plants. Osmotic stress pronounced the lipid peroxidation and superoxide anions formation in control plants as compared to endophyte and SA-treated plants. The endogenous SA contents were significantly higher in pepper plants treated with endophyte and SA under osmotic stress as compared to control.

Conclusion: Endophytic fungal symbiosis and exogenous SA application can help the plants to relieve the adverse effects of osmotic stress by decreasing losses in biomass as compared to non-inoculated plants. These findings suggest that SA application positively impact microbial colonization while in combination, it reprograms the plant growth under various intervals of drought stress. Such symbiotic strategy can be useful for expanding agriculture production in drought prone lands.
\end{abstract}

Keywords: Penicillium resedanum LK6, Osmotic stress, Salicylic acid, Antioxidants, Biomass recovery, Capsicum annuum L

\section{Background}

Water-deficient or drought stress conditions can drastically hinder the crop growth and yield. Exposure to extreme conditions brings changes inside plant tissues at ionic/osmotic, phytohormonal and secondary metabolites levels [1]. Continuous waves of drought cause an imbalance in the osmotic potential of the plant tissues, thus, inducing the synthesis of reactive oxygen species (ROS) [2]. To maintain the cellular redox potential and buffer the

\footnotetext{
* Correspondence: ijlee@knu.ac.kr

${ }^{2}$ School of Applied Biosciences, Kyungpook National University, Daegu, Republic of Korea

Full list of author information is available at the end of the article
}

negative effects of ROS, plant produce antioxidants like reduced glutathione (GSH), total polyphenols, catalase (CAT), peroxidase (POD) and polyphenol oxidase (PPO) etc [3]. Plants tend to accumulate higher antioxidants to avoid cellular damage. Additionally, the plant hormonal apparatus is activated to transduce stress signals during altered osmotic potential. Endogenous salicylic acid (SA) is known to develop defencerelated responses during biotic stress $[4,5]$ while exogenous application of SA has mostly showed abiotic stress tolerance for example, heat stress in mustard [6], chilling in maize [7], salinity in wheat [8] and drought in wheat and sunflower $[9,10]$. Exogenous

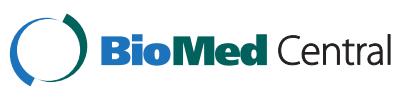


SA increase shoots length, flowering and yield in various crop plants [4-10]. During pathogenic attack, the endogenous SA in plants is often accumulated whilst the systemic acquired resistance (SAR) is initiated which involve synthesis of pathogenesisrelated $(P R)$ proteins [3]. Conversely, during interaction with mutualistic plant growth promoting microorganism, it doesn't involve the synthesis of $P R$ protein, thus establishing induced systemic resistance (ISR) $[11,12]$. In spite of the key role of SA in plant's defence, its function during endophyte-association has received little attention [13].

Endophytic fungi, residing in the root tissues can play pivotal role in host-plant growth by influencing mineral composition, plant hormonal balance, chemical composition of root exudates, soil structure and plant protection against biotic and abiotic stresses [14-16]. Previous studies have shown that endophytic fungal association can significantly increase plant biomass and growth [14-18]. Studies have also elaborated the beneficial effects of endophytic fungi on the growth responses of host-plants under various stress conditions [15-18]. In present study, we isolated an endophytic fungus, Penicillium resedanum LK6 from the roots of drought-stressed Capsicum annuum (pepper) plants. The endophyte was found to produce various biologically active gibberellins detected in the pure culture through chromatographic techniques and advance spectroscopic analysis (unpublished results). Previous studies also show that some strains of Penicillium endophytes can produce gibberellins [17]. Redman et al. [16] and Khan et al. [17] have previously shown that phytohormones producing endophytes/fungi can ameliorate the negative impacts of salinity and drought.

Gibberellins producing fungal endophytes have been envisaged to increase host-plant resistance against salinity, drought, and heat stresses $[16,17]$ however, these are least known for their symbiotic impacts on endogenous and exogenous SA during abiotic stress. Previously, Herrera-Medina et al. [11] explained the influence of exogenous SA on root colonization but it was mostly restricted to arbuscular mycorrhizal fungi [12]. A similar study was reported by $\mathrm{Li}$ et al [19] in which the effect of exogenous SA on the colonization of arbuscular mycorrhizal fungi Glomus mosseae and growth of Avena nuda resistance under $\mathrm{NO}_{2}$ exposure were assessed. However, the interaction of exogenous SA and endophyte association with $C$. annuum plants during stress is still poorly understood and unexplored. In present study, it was aimed to understand the co-synergism of SA with endophytic fungus (Penicillium resedanum LK6) and its effects on plant biomass recovery under polyethylene glycol (PEG) induced osmotic stress (2, 4 and 8 days).

\section{Methods}

Growth of endophytic fungus - Penicillium resedanum LK6 Approximately, 200 root pieces were collected from C. annuum plants growing in water deficient conditions (soil water potential $41.23 \mathrm{hPa}$ ). The root pieces were surface sterilized with $2.5 \%$ sodium hypochlorite (30 min in shaking incubator at $120 \mathrm{rpm}$ ) and washed with autoclaved distilled water (DW) to remove the contaminants, rhizobacteria and superficial fungi. The pepper root pieces (about $0.5 \mathrm{~cm}$ ) were kept in petri-plates containing Hagem medium $\left(0.05 \% \mathrm{NH}_{4} \mathrm{Cl}, 0.1 \% \mathrm{FeCl}_{3}\right.$, $0.05 \% \mathrm{KH}_{2} \mathrm{PO}_{4}, 0.5 \%$ glucose, $0.05 \% \mathrm{MgSO}_{4} .7 \mathrm{H}_{2} \mathrm{O}, 1.5 \%$ agar and $80 \mathrm{ppm}$ streptomycin; $\mathrm{pH} 5.6 \pm 0.1)$. The sterilized roots pieces were imprinted to ensure the effectiveness of sterilization process Redman et al. [16]. The emerging fungal spots from the root pieces were isolated and transferred to Potato Dextrose Agar (PDA) medium under aseptic conditions. Among isolated endophytes, a bioactive strain was selected through screening bioassays using dwarf mutant and normal cultivars of Oryza sativa. The endophyte was identified by DNA extraction, PCR techniques, sequencing and phylogenetic analysis of Internal Transcribed Spacer [ITS-1 (5'TCC GTA GGT GAA CCT GCG G-3') and ITS-4 (5'TCC TCC GCT TAT TGA TAT GC-3')] with the method previously described by Redman et al. [16] and Khan et al. [17]. The sequence of the endophyte ( $P$. resedanum) was submitted to GenBank and was given accession no. JX111908. The endophyte was inoculated in Czapek broth (1\% peptone, $1 \%$ glucose, $0.001 \% \mathrm{FeSO}_{4} .7 \mathrm{H}_{2} \mathrm{O}, 0.05 \% \mathrm{MgSO}_{4} .7 \mathrm{H}_{2} \mathrm{O}, 0.05 \% \mathrm{KCl}$; $\mathrm{pH} 7.3 \pm 0.2)$ and incubated for 10 days at $28^{\circ} \mathrm{C}$ under shaking $(150 \mathrm{rpm})$ conditions to undertake further experiments $[17,18]$.

\section{C. annuum growth with endophyte}

The C. апnиum seeds were sterilized with $2.5 \%$ sodium hypochlorite for $30 \mathrm{~min}$, and rinsed with autoclaved DW. Seeds were incubated in darkness for $24 \mathrm{~h}$ to obtain equally germination. The pregerminated seeds were cultivated in autoclaved pots $\left(121^{\circ} \mathrm{C}\right.$ for $15 \mathrm{~min}$; two times; $\left.10 \times 5 \mathrm{~cm}\right)$ with substrate (peat: perlite: vermiculite - 1:1:1 by volume). The endophyte was cultured in Czapek broth containing conidia (20 ml with 25 propagules/pot) and added to substrate as described previsouly [16-18]. The control plants only received $20 \mathrm{ml} /$ pot of endophyte-free Czapek broth. Thus, pre-germinated pepper seeds and endophyte were grown together for three weeks in the growth chamber (day/night cycle: $14 \mathrm{~h} ; 28^{\circ} \mathrm{C} / 10 \mathrm{~h} ; 25^{\circ} \mathrm{C}$; relative humidity $60-70 \%$; light intensity $1000 \mu \mathrm{Em}^{-2-} \mathrm{s}$ Natrium lamps) irrigated with distilled water. 
Drought stress, endophyte association and SA treatments The experiment was conducted with a completely randomized block design. Salicylic acid $\left(\mathrm{SA}-10^{-6} \mathrm{M}\right)$ was exogenously applied to pepper plants. The treatments included (i) control, (ii) control plants under drought stress, (iii) plants with endophyte (EA), (iv) EA plants under stress, (v) SA-treated plants, (vi) SA-treated plants under stress, (vii) SA and endophyte-infected plants and (viii) SA and endophyte-associated plants under stress $(\mathrm{SA}+\mathrm{EA})$. Each treatment contained 18 plants and the experiment was repeated three times. Drought stress was initiated by exposing plants to $15 \%$ polyethylene glycol (PEG 10,000 MW; -3.02 MPa osmotic potential) for 2,4 and 8 days. The growth parameters i.e. shoot length and fresh weights were measured at harvest while chlorophyll content of leaves was measured by chlorophyll meter (SPAD-502 Minolta, Japan). All readings were taken in triplicate.

The effect on the plant biomass was measured after endophyte and SA treatments under different stress regimes [18]. The biomass gained/lost in endophyteinoculated and non-inoculated plants were compared by using this formula:

$$
\text { Biomass increment: }{ }^{\mathrm{DW}(E+)-\mathrm{DW}(E-)} /{ }^{\mathrm{DW}(E-)} \times 100 \text {. }
$$

DW is the dry weight while E+ and E- are plants with or without endophyte infestation respectively.

\section{Determination of electrolytic leakage}

Electrolytic leakage was determined according to the method of Liu et al. [20]. Briefly, fresh leaf samples (200 mg) were cut into $5 \mathrm{~mm}$ small pieces length and placed in test tubes containing $10 \mathrm{ml} \mathrm{DW}$. The preliminary electrical conductivity $\left(\mathrm{EC}_{1}\right)$ was measured after the tubes were kept in water bath at $25^{\circ} \mathrm{C}$ for one hour. The samples were autoclaved at $121^{\circ} \mathrm{C}$ for $20 \mathrm{~min}$ to completely kill the tissues and release all electrolytes from leaf tissues. When the samples were cooled down to $25^{\circ} \mathrm{C}$, final electrical conductivity $\left(\mathrm{EC}_{2}\right)$ was measured. The electrolyte leakage (EL) was estimated using formula: $\mathrm{EL}=\mathrm{EC}_{1} / \mathrm{EC}_{2}$.

\section{Microscopic analysis and colonization}

Plant roots infected with fungal endophyte were sectioned and treated with sodium hypochlorite (2.5\%) for $10 \mathrm{~min}$ for clarification. Latter, it was treated with $\mathrm{KOH}(20 \%)$ for $24 \mathrm{~h}$ which was extensively rinsed with autoclaved DW. The root pieces were acidified with $\mathrm{HCl}$ (10\%); stained for $24 \mathrm{~h}$ using tryptophan blue $(0.8 \%)$ and lactic acid (95\%). At the end, the root pieces were distained in lactic acid for $24 \mathrm{~h}$. The endophytic colonization in roots pieces was assessed through light microscope (Stemi SV 11 Apo, Carl Zeiss). The rate of colonization was determined according to the method of Kumar and Hyde [21].

\section{Determination of antioxidants}

To determine reduced glutathione (GSH), leaves tissues $(100 \mathrm{mg})$ of all the treated pepper plant samples were ground in $3 \mathrm{ml} \mathrm{5 \%} \mathrm{(v/v)} \mathrm{trichloroacetic} \mathrm{acid} \mathrm{using} \mathrm{chilled}$ mortar and pestle. The homogenate was obtained through centrifugation (at $15000 \mathrm{rpm}$ for $15 \mathrm{~min}$ at $4^{\circ} \mathrm{C}$ ). The homogenate obtained was analysed for reduced glutathione (GSH) activity as described by Ellman [22]. The reaction mixture comprised of sample supernatant $(0.1 \mathrm{ml})$, monosodium phosphate $(3.0 \mathrm{ml} ; 150 \mathrm{mM}$ $\left.\mathrm{NaH}_{2} \mathrm{PO}_{4} ; \mathrm{pH} 7.4\right)$ and Ellman's reagent $(0.5 \mathrm{ml})$. The mixture was incubated at $30^{\circ} \mathrm{C}$ for $5 \mathrm{~min}$. Absorbance was determined at $412 \mathrm{~nm}$ and the GSH activity was calculated by a standard curve.

Total polyphenol content was determined by the Folin-Ciocalteau method as mentioned by Kumazawa et al. [23]. Plant tissues (100 mg) were ground with $80 \%$ ethanol and the resultant extracts $(0.5 \mathrm{ml})$ were mixed with Folin-Ciocalteau reagent $(0.5 \mathrm{ml})$ and $10 \% \mathrm{Na}_{2} \mathrm{CO}_{3}$ $(0.5 \mathrm{ml})$. The absorbance of the reaction mixture was measured at $760 \mathrm{~nm}$ after $1 \mathrm{~h}$ incubation at room temperature. Total polyphenol content was expressed as micro g/mg (gallic acid equivalents).

The detection of superoxide anion $\left(\mathrm{O}_{2}^{-}\right)$was based on its ability to reduce nitro blue tetrazolium (NBT) as performed by Doke [24]. Treated plant tissues (100 mg) were cut into $1 \mathrm{~mm}^{2}$ pieces and immediately immersed in $10 \mathrm{mM}$ phosphate buffer $(\mathrm{pH} 7.8)$, containing NBT $(0.05 \%(\mathrm{w} / \mathrm{v}))$ and $10 \mathrm{mM} \mathrm{NaN}_{3}$. The reaction mixture was left for incubation till one hour at room temperature. The reaction mixture was heated at $85 \pm 2^{\circ} \mathrm{C}$ for $15 \mathrm{~min}$ and cooled quickly to $0^{\circ} \mathrm{C}$. The absorbance was measured at $580 \mathrm{~nm}$. The $\mathrm{O}_{2}^{-}$content was expressed as an increase of absorbance / $0.1 \mathrm{~g}$ dry weight.

The extent of lipid peroxidation was determined by the method of Ohkawa et al. [25]. The optical density of the resulting light pink colour was recorded at $532 \mathrm{~nm}$. Tetramethoxypropane was used as an external standard. The level of lipid peroxides was expressed as micro moles of malondialdehyde (MDA) formed/g tissue weight.

\section{Enzymatic analysis}

All treated plant's leaves (200 mg) were homogenized in $50 \mathrm{mM}$ Tris- $\mathrm{HCl}$ buffer $(\mathrm{pH} 7.0)$ composed of $3 \mathrm{mM}$ $\mathrm{MgCl}_{2}, 1 \mathrm{mM}$ EDTA and 1.0\% PVP and then centrifuged $\left(15,000 \mathrm{rpm}\right.$ for $15 \mathrm{~min}$ at $\left.2^{\circ} \mathrm{C}\right)$. The supernatant was used for enzymatic analysis. All parameters were expressed as activity per mg protein. Total proteins were determined according to Bradford [26] method. 
Catalase (EC 1.11.1.6) activity was measured as describe by Aebi [27]. The crude enzyme supernatant was treated with $0.2 \mathrm{M} \mathrm{H}_{2} \mathrm{O}_{2}(0.5 \mathrm{ml})$ in $10 \mathrm{mM}$ phosphate buffer ( $\mathrm{pH}$ 7.0). The enzymatic activity was determined by the decrease in absorbance of $\mathrm{H}_{2} \mathrm{O}_{2}$ at $240 \mathrm{~nm}$. The one unit of catalase is given as $\mu$ g of $\mathrm{H}_{2} \mathrm{O}_{2}$ released mg protein $\mathrm{min}^{-1}$. Peroxidase (EC 1.11.1.7) and polyphenol oxidase (EC 1.14.18.1) activities were measured as described by Kar and Mishra et al. [28] with a little modification. The pepper leaf samples (200 mg) were homogenized with phosphate buffer $\mathrm{pH} 6.8(0.1 \mathrm{M})$ and centrifuged $\left(2^{\circ} \mathrm{C}\right.$ for $15 \mathrm{~min}$ at $\left.17,000 \mathrm{rpm}\right)$. The clear supernatant was obtained which was analysed for enzymatic activity. The reaction mixture of peroxidase activity composed of $0.1 \mathrm{M}$ phosphate buffer ( $\mathrm{pH} 6.8$ ), pyrogallol $(50 \mu \mathrm{l}), \mathrm{H}_{2} \mathrm{O}_{2}(50 \mu \mathrm{l})$ and enzyme extract $(0.1 \mathrm{ml})$. After incubation $\left(5 \mathrm{~min}\right.$ at $\left.25^{\circ} \mathrm{C}\right)$, the reaction was stopped by adding $5 \%(\mathrm{v} / \mathrm{v}) \mathrm{H}_{2} \mathrm{SO}_{4}(0.5 \mathrm{ml})$. The amount of purpurogallin synthesized during the reaction was measured by the absorbance at $420 \mathrm{~nm}$. The same assay mixture, used for peroxidase (without $\mathrm{H}_{2} \mathrm{O}_{2}$ ), was measured for the activity of polyphenol oxidase. The absorbance of purpurogallin formed was read at $420 \mathrm{~nm}$. One unit of peroxidase and polyphenol oxidase was defined as an increase of 0.1 units of absorbance.

\section{Endogenous salicylic acid analysis}

SA was extracted and quantified as described previously by Seskar et al. [29]. The freeze-dried leaf tissues ( $0.4 \mathrm{~g})$ of all treated samples were grinded to powder. The powder was sequentially extracted with 90 and $100 \%$ methanol by centrifuging (at $15,000 \mathrm{rpm}$ and $4^{\circ} \mathrm{C}$ ). Both the extraction steps were repeated four times until the sample decoloured. The combined methanol extracts were vacuum-dried. Dry pellets were re-suspended in $5 \%$ trichloroacetic acid $(2.5 \mathrm{ml})$ while the supernatant was partitioned with ethyl acetate: cyclopentane: isopropanol (100:99:1, v/v). The organic layer containing free SA was transferred to a $4 \mathrm{ml}$ vial and dried with nitrogen gas. The dry SA was rigorously suspended in $1 \mathrm{ml}$ of $70 \%$ methanol. High Performance Liquid Chromatography (HPLC) analysis were carried out on Shimadzu coupled with fluorescence detector (Shimdzu RF-10AXL, excitation and emission 305-365 nm respectively) fitted with C18 reverse-phase HPLC column (HP hypersil ODS, particle size $5 \mu \mathrm{m}$, pore size $120 \AA$ Waters) (Additional file 1: Table S1). The flow rate was $1.0 \mathrm{ml} / \mathrm{min}$. The experiment was repeated three times.

\section{Statistical analysis}

The eight different treatments comprised of eighteen plants per treatment while the experiments were performed in triplicate. The mean, standard error and the graphical representation was done using Graph Pad
Prism software (version 5.0, San Diego, California USA). To identify significant effects between the treatments and control with or without stress conditions and endophyte, we used Duncan's multiple range tests (DMRT) on Statistic Analysis System (SAS 9.1, USA).

\section{Results}

\section{Co-synergism of endophyte and SA in plant biomass recovery under stress}

The germinated pepper seeds were grown together with fungal endophyte $P$. resedanum (culture filtrate and mycelial propagules). After one week of endophyte association, the growth promoting effects were visible as compared to non-inoculated control plants. The endophyte-infected plants had higher growth rate and plant length than the control plants (Figure 1A). Similarly, when pepper plants were exposed to shortterm drought stress for two, four and eight days, the shoot length was significantly reduced in noninfected control plants as compared to endophyteelicited plants (Figure $1 \mathrm{~B}$ and 2). With the increasing duration of drought stress, the plant height and metabolism reduced however, this was more pronounced in control than endophyte-inoculated plants. A similar growth dynamics was also shown by the exogenous application of SA with or without exposure to drought stress conditions (Figure 2). The endophyte-infected plants when applied with SA (with or without stress) resulted in significantly higher shoot length as compared to sole SA and control. Contrarily, the shoot lengths of plants inoculated with endophyte and treated with SA (SA+EA) and endophyteassociated (EA) plants were not significantly different from each other. It was observed that the non-inoculated plants without SA had significantly lower shoot lengths than the other three treatments i.e. EA, SA and SA+EA. Overall, the effect on shoot growth in EA and SA plants were not significantly different from each other. However, combination of SA+EA treatment exhibited significantly higher plant length as compared to other treatments.

The chlorophyll contents was higher in endophyteinfected plants than in non-infected plants. The droughtstress treated plants had significantly lower level of chlorophyll in non-inoculated plants whilst this was significantly higher in SA, EA and SA+EA plants during stress and normal growth conditions (NST). In control plants, the chlorphyl content was decreasing with the gradual exposure to drought (Figure 2). Conversely, in EA, SA and SA+EA plants, this trend was increasing with or without drought stress. It was significantly higher in $\mathrm{SA}+\mathrm{EA}$ plants exposed to maximum duration of water deficient conditions. Beside this, we also observed that the photosynthesis rate was significantly higher in EA, SA and $\mathrm{SA}+\mathrm{EA}$ plants. 


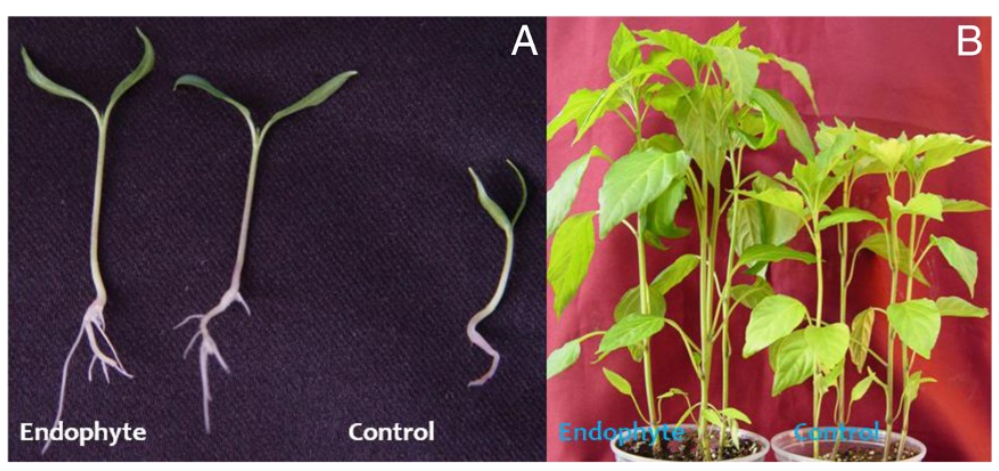

Figure 1 Endophyte $P$. resedanum inoculated pepper (Capsicum annuum L.) plants growth after one week (A) and three weeks before stress (B). Representative photo of pepper seedlings (18 per treatment) inoculated with or without endophytic fungi.

The shoot length was $17.4,13.3$ and $23.3 \%$ higher in EA, SA and SA+EA treatments as compared to control after two days of stress. Similarly, after 4 and 8 days of stress, the shoot length increased 15.2, $10.8,19.7 \%$ and $12.2,9.1,19.2 \%$ in EA, SA and SA+EA treatments respectively as compared to control (Figure 3). The biomass gains were prominent in the EA and SA+EA. During drought stress, the biomass loss was more prominent in control plants while our results did not shown significant difference between SA and EA plants (Figure 2).

Similarly, the plant biomass improvement during $\mathrm{EA}$ and $\mathrm{SA}+\mathrm{EA}$ was also varified by the reduced electrolytic leakage (EL) in plants under stress. The results showed that EL was significantly higher in the non-inoculated control plants treated with 2,4 and 8 days of drought. It was highly significant $(P<0.001)$ in control after 8 days of stress (Figure 3$)$. In comparison to sole SA-treated plants, the EL was lower than EA and SA+EA plants (Figure 3). The results suggest that the increased electrolytes influx represent higher tissue damages inside plants while this has been counteracted by the presence of endophyte with or without stress conditions.

The microscopic images showed the active association and habitation of $P$. resedanum inside the pepper plant's root. The non-infected control plant's roots were without any fungal association (Figure 4). The epidermal and cortex cellular region had no fungal infection. Contrarily, the microsclerotium of endophyte was seen in the inner cortex regions of the EA plant roots under normal growth conditions after one week of inoculation. However, endophyte colonization increased inside root with the passage of time and stress period. In SA+EA plants after 8 days of droughts stress, the rate of colonization was higher than the EA plants, suggesting that SA can also play an essential role in symbiotic microbial association (Figure 4).
Antioxidant's modulation during stress with $P$. resedanum and SA

The results of antioxidant activities reveal stress modulation in pepper plants in the presence of endophyte as well as SA+endophyte under drought stress. The oxidative stress was promulgated by the imbalance in cellular water potential in control. In non-inoculated control, the total polyphenols were significantly lower than that of EA, SA and SA+EA treated plants. Though, the EA and SA plants had almost similar level of total polyphenol however in SA+EA plants, it was significantly higher. With immediate advent of stress conditions for two days, the total polyphenol level dropped down in noninoculated plants as compared to other treatments like SA, EA and SA+EA treated plants. After 2 days of stress, endophyte-infested and SA treated plants have significantly higher total polyphenol levels as compared to sole EA and SA treated plants (Figure 5). Similarly, the increased osmotic stress in pepper further deteriorated the total polyphenol levels in control plants under 4 and 8 days of drought stress as compared to EA, SA and SA+EA plants. During high osmotic stress, the endophyteassociated plants maintained the total polyphenol level. We observed no significant different between EA, SA and $\mathrm{SA}+\mathrm{EA}$ treated plants after exposure to 8 days of stress period.

Reduced glutathione (GSH) contents were significantly lower in control plants as compared to EA and $\mathrm{SA}+\mathrm{EA}$. The highest level of GSH formation was observed in SA+EA plants than other treatments. Upon osmotic stress, the GSH level reduced sharply in control plants as compared to other treatments (Figure 5). At $4^{\text {th }}$ and $8^{\text {th }}$ day of stress, the control and SA treated plant's GSH level was lower than that of the EA and SA+EA plants. On $8^{\text {th }}$ day of stress, EA, $\mathrm{SA}$ and $\mathrm{SA}+\mathrm{EA}$ plants were not significantly different in GSH level as compared to control plants. Thus, endophyte-association seems to have counteracted the stress in the presence of SA application. 

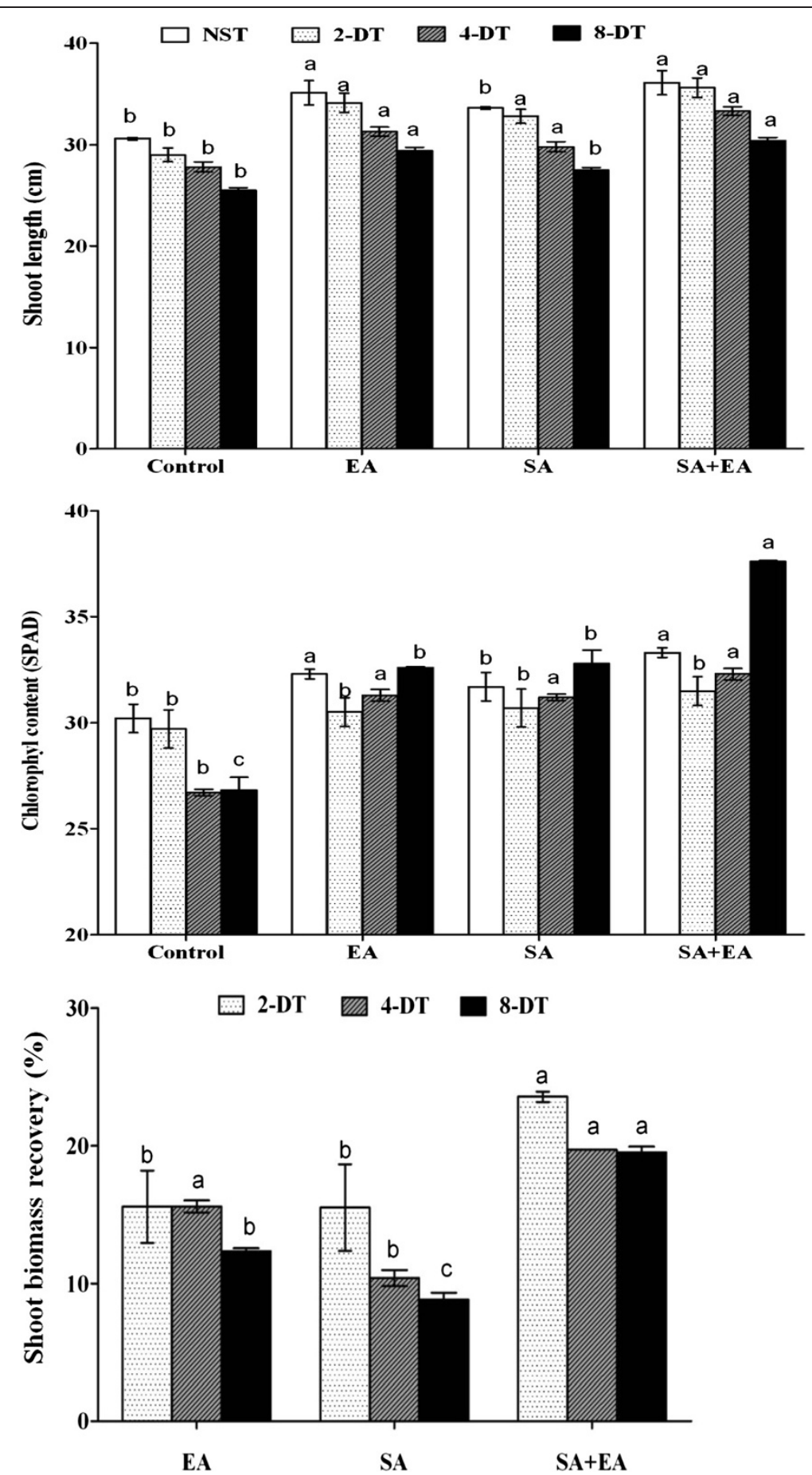

Figure 2 Effects of endophyte $P$. resedanum association on the shoot length, chlorophyll content and shoot biomass recovery in various treatments after exposure to osmotic stress. The control plants were treated with distilled water. EA plants were infected with P. resedanum; SA plants treated with $10^{-6} \mathrm{M} \mathrm{SA}$, while SA+EA plants had endophytic-fungal association and treated with SA. 2-DT, 4-DT and 8-DT represent the osmotic stress induced by PEG for 2, 4 and 8 days respectively; NST (not stressed treatment). The different letter (s) in each treatment showed significant difference $(P<0.05)$ as evaluated by DMRT.

The extent of lipid peroxidation (MDA content) was significantly regulated during the presence of endophyticfungal association and SA application. The EA and SA+EA plant had lower level of MDA formation as compared control plants. SA and control plants, on the other hand, had no significant difference in MDA production under normal growth conditions. During osmotic stress, the MDA level in control plants increased abruptly from 2 to 


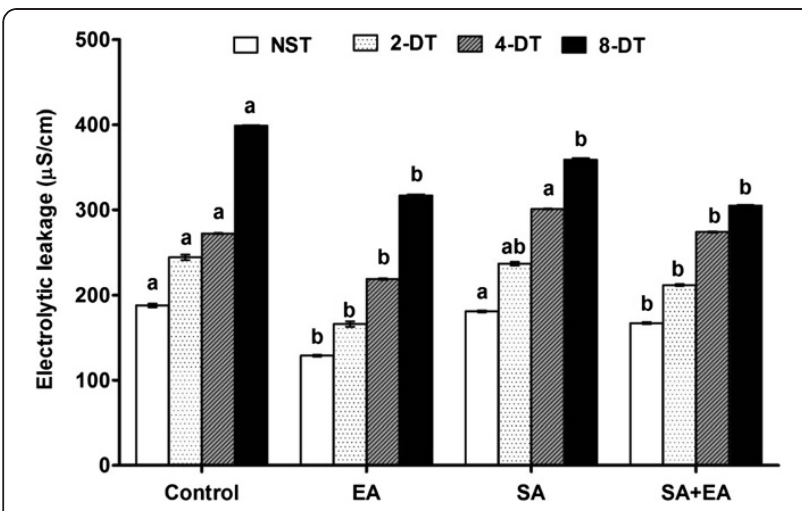

Figure 3 Effect of endophyte symbiosis on the electrolytic release during stress. $E A=$ infected with $P$. resedanum; $S A=$ treated with $S A ; S A+E A$ = endophytic-fungal associated plants treated with SA. NST (not stressed treatment), 2-DT, 4-DT and 8-DT represent drought stress period of 2, 4 and 8 days respectively.

8 days stress period. Conversely, this trend was significantly lower in SA, EA and SA+EA plants. Though, the MDA levels were high in SA treatments at the $8^{\text {th }}$ day of stress but this was significantly lower than that of control plants (Figure 5). Results suggest that the endophyte presence has counteracted drought stress by minimizing lipid peroxidation.

Supper oxide anions $\left(\mathrm{O}_{2}-\right)$ were not significantly different between EA and SA plants. $\mathrm{O}_{2}^{-}$levels were higher in control plants under normal conditions. After the exposure to stress conditions ( 2 and 4 days), the $\mathrm{O}_{2}^{-}$formation was significantly high in control plants as compared to EA, SA and EA+SA plants. After $8^{\text {th }}$ day of stress, the $\mathrm{O}_{2}^{-}$levels were high in control and SA as compared to $\mathrm{EA}$ and $\mathrm{SA}+\mathrm{EA}$ plants (Figure 5).

\section{Enzymatic regulation by endophyte and SA under stress}

Enzymatic activities were significantly regulated during EA, SA and SA+EA. In catalase activity (CAT), it was significantly higher in EA and SA+EA plants while it was not significantly different between SA and control. In exposure to 2 days stress, the catalase activity was significantly activated in endophytic-associated plants as compared to control plants, SA and SA+EA plants. This activation trend was followed by $\mathrm{SA}+\mathrm{EA}$ and SA plants respectively (Figure 6). In 4 and 8 days of stress, the catalase activity gradually reduced in EA plants whilst in SA and SA+EA it increased sharply. The catalase activity was significantly higher in $\mathrm{SA}+\mathrm{EA}$ plants as compared to other treatments and control plants under maximum period of stress.

Peroxidase (POD) activities were significantly reduced in control plants with or without stress conditions as compared to other SA, EA and SA+EA plants. Under normal growth conditions, POD activity was significantly higher in EA and SA+EA plants as compared to SA
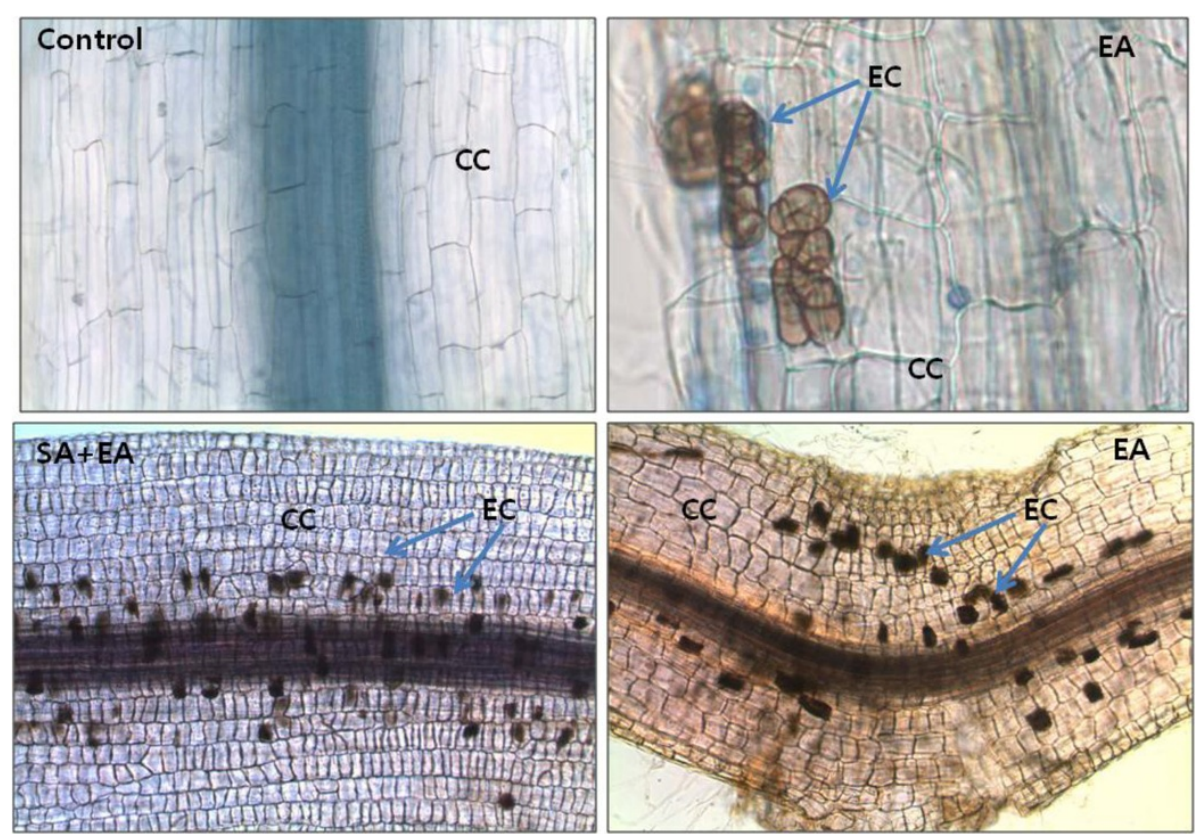

Figure 4 Light micrographs of endophyte $P$. resedanum - associated with host plant's root. (Control) shows the light microscopic image of endophyte-free control plants (two weeks old). Bar $=200 \mu \mathrm{m}$. (EA) pepper root infected with $P$. resedanum after one week of inoculation. Microsclerotiums in the form of yeast-like cells were extensively colonized in the middle and inner cortex of the root. Bar $=40 \mu \mathrm{m}$; Bar $=100 \mu \mathrm{m}$. $(\mathrm{SA}+\mathrm{EA})$ presence of brownish yeast-like cells pericycle regions of the roots. Bar $=100 \mu \mathrm{m}$. The root samples were stain with tryptophan blue $(0.8 \%)$. In the micrographs, CC = cortex cells; EC = endophyte cell. 

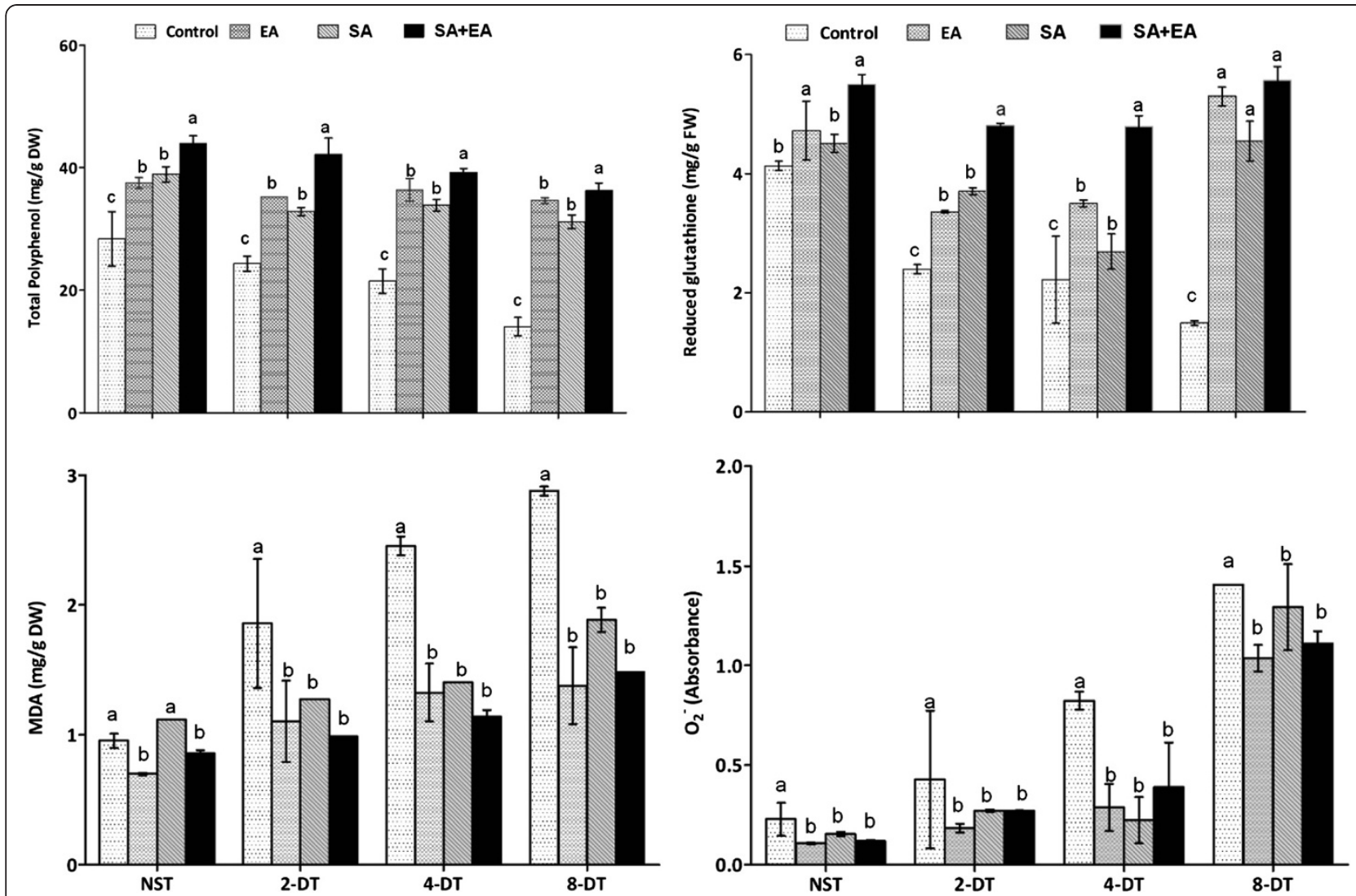

Figure 5 Influence of drought stress on the antioxidants activities of the pepper plants inoculated with or without endophyte. MDA refers to extent of lipid peroxidation; $\mathrm{O}_{2}^{-}$refers to superoxide anion. $\mathrm{EA}=$ infected with $P$. resedanum; $\mathrm{SA}=$ treated with $\mathrm{SA} ; \mathrm{SA}+\mathrm{EA}=$ endophytic fungal associated plants treated with SA. NST, 2-DT, 4-DT and 8-DT represent non-stressed, 2, 4 and 8 days drought stressed plants respectively. The different letter(s) in each stress period showed significant difference $(P<0.05)$ as evaluated by DMRT.

plants (Figure 6). Upon exposure to osmotic stress for 2, 4 and 8 days, the POD activity was significantly higher in EA plants than SA and SA+EA plants and control plants. However, SA+EA plants had higher POD activity as compared to SA and control plants.

Polyphenol oxidase (PPO) activities were significantly reduced in control pepper plants. PPO activities increased in a dose dependent manner in EA plants with or without stress conditions. It was significantly higher in EA plants after 8 days stress (Figure 6). In SA treatments, PPO response with or without stress conditions was irregular. Although, PPO activity was comparatively lesser in SA+EA plants, it followed the same trend as we observed in EA plants.

\section{$P$. resedanum association and SA-dependent responses under abiotic stress}

We also assessed the effect of endophytic elicitation with or without the treatment of SA on endogenous SA level. The results showed that $\mathrm{SA}$ was significantly low in non-stressed control. However, the stress periods has increased the endogenous SA levels (Figure 7). Similarly, in endophyte-associated plants, the endogenous SA was significantly higher than control under normal growth conditions. While after 2 days stress, its level insignificantly increased. The 4 and 8 days stress significantly increased SA contents in EA plants. This level was significantly higher than that of control and SA treated plants. In sole SA treatments, the plant synthesized low level of SA without any stress. However, upon 2 and 4 days stress, the SA level increased significantly while after 8 days, it decreased. In case of SA+EA plants, the endogenous SA followed the same trend as we noticed in sole SA treatments, however, the quantity of SA synthesized was significantly higher during similar conditions (Figure 7). The overall SA biosynthesis pathway activation in sole SA was lower than EA and SA+EA plants. The EA and SA+EA plants have significantly activated endogenous SA biosynthesis with or without stress conditions.

\section{Discussion}

Endophyte-association helps in biomass recovery

The results of the present study support and give additional information on the mechanism of endophyte's 

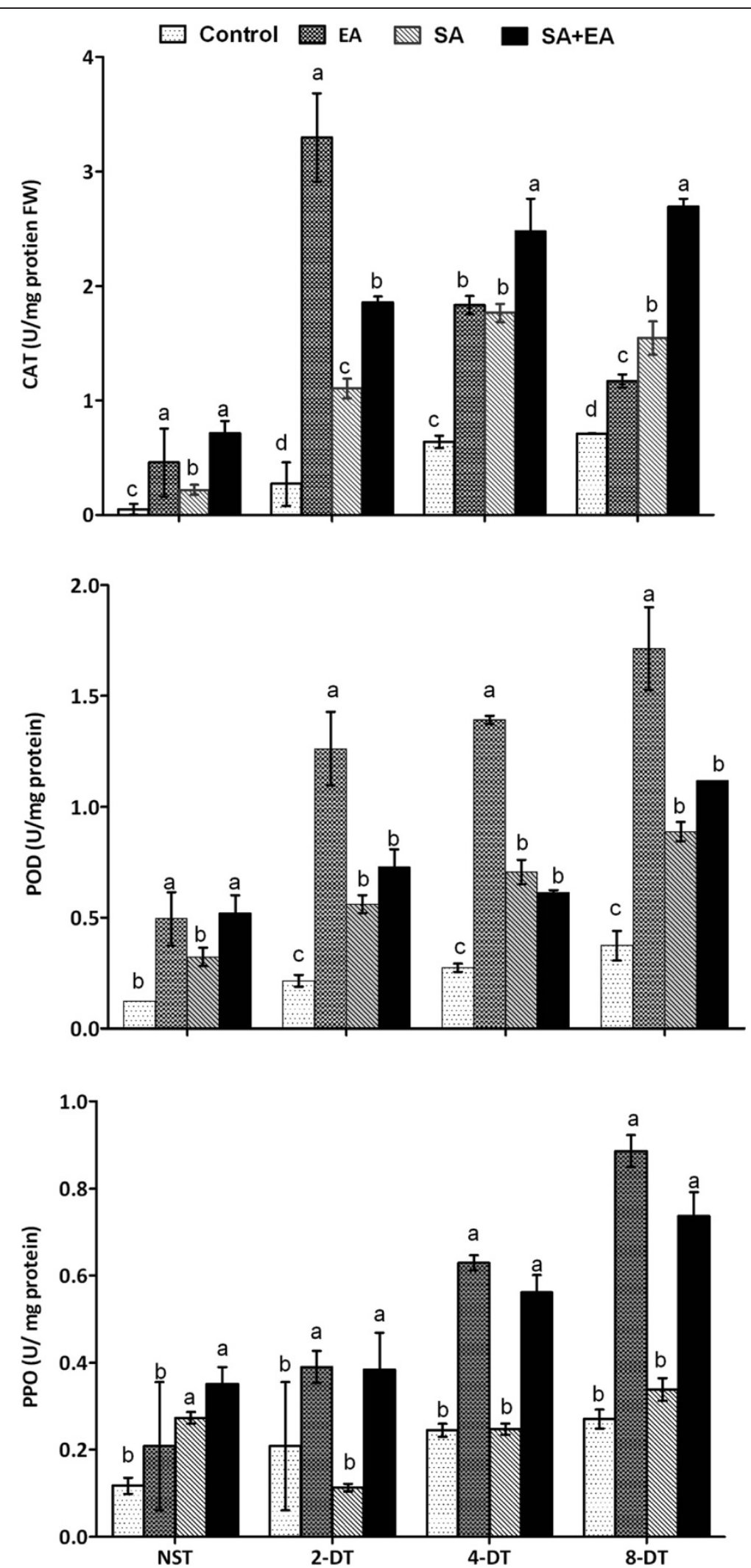

Figure 6 (See legend on next page.) 
(See figure on previous page.)

Figure 6 Enzymatic activities of endophyte, SA and endophyte with SA treated plants during drought stress. $C A T=$ catalase; $P O D=$ peroxidase; PPO = polyphenol peroxidase. EA = infested with $P$. resedanum; SA = treated with SA; SA+EA = endophytic fungal associated plants treated with SA. NST, 2-DT, 4-DT and 8-DT represent non-stressed, 2, 4 and 8 days drought stressed plants respectively. The different letter(s) in each stress period showed significant difference $(P<0.05)$ as evaluated by DMRT.

ameliorative potential during abiotic stress to crop plant. The results revealed that endophyte-association rescued growth of pepper plants during stress by increasing shoot length. Plant-fungus relationship has been proclaimed a pivotal source for plant growth and development [30,31]. Endophytic fungi have been regarded as plant protectant and growth regulator during normal and extreme environmental conditions [15-20,31-33]. Various novel endophytic fungal species like Piriformospora indica, Neotyphodium sp., Curvularia protuberate, and Colletotrichum sp. etc $[19,20,31,32,34]$ have been known to improve plant growth during abiotic stress conditions. Penicillium species have been known as a vital source for bioactive secondary metabolites [35]. Some strains of this genus also produce plant growth regulators like gibberellins, auxin, etc $[17,19,33,36]$.

Gibberellins producing fungal genes cluster have been recently identified for Phaeosphaeria sp. L487 [37], Gibberella fujikuroi, Sphaceloma manihoticola [38] etc. Previous studies have shown that Penicillium citrinum [39], P. paxilli [40], P. funiculosum [17] produces gibberellins. It suggests the existence of GAs gene cluster in Penicillium spp; hence, needs further genomic analyses at transcriptomics levels. In endophyte-host symbioses, consequences and interaction of secondary metabolites may be a contribution of the fungal endophyte to its host-plant to establish a mutualistic relationship $[32,41]$. Though, this process is very slow and the

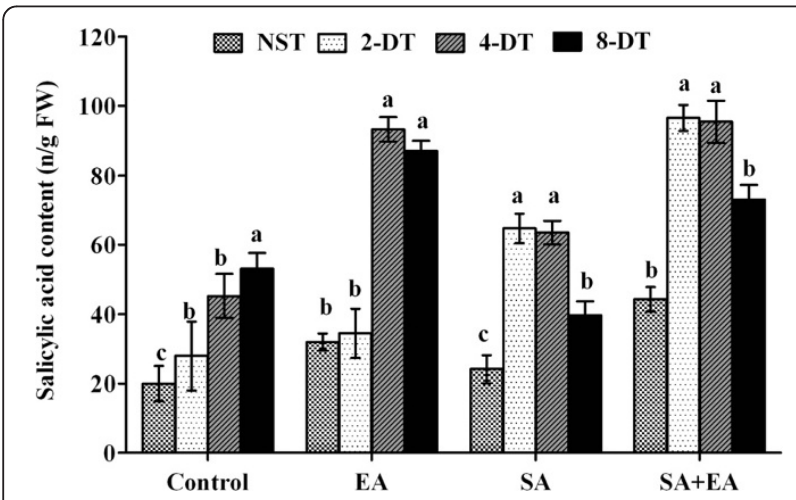

Figure 7 Endogenous salicylic acid (SA) synthesis of pepper plants inoculated with or without $P$. resedanum under osmotic stress and normal growth conditions. $E A=$ infected with $P$. resedanum; $S A=$ treated with $S A ; S A+E A=$ endophytic fungal associated plants treated with SA. NST, 2-DT, 4-DT and 8-DT represent non-stressed, 2, 4 and 8 days drought stressed plants respectively. The different letter (s) in each stress period showed significant difference $(P<0.05)$ as evaluated by DMRT. quantities of metabolites are very minute depending upon host and endophyte, but one way or the other, this barter trade always supports the host to counteract stress periods.

The phytohormones synthesis potential gives additional benefits to the host plants in mitigating the adverse affects of extreme environmental conditions salinity, drought and temperature stress as shown by Redman et al. [16], Khan et al. [17] and Hamilton and Bauerle [31]. Plants treated with the culture filtrate and propagules of endophytes are often healthier than endophyte-free ones [19,32]. Indeed, the endophyte-associations have enhanced biomass of barley [16], tomato [15], soybean [17] and rice [16] plants under various abiotic stress conditions like salinity, drought and high temperature. Pepper plants are adversely affected by abiotic stresses which retard their yield. It was observed that $P$. resedanum -associated plants had higher shoot length, chlorophyll content, and photosynthesis rate and low electrolytic leakages as compared to noninoculated control. The non-inoculated plants, on the other hand, deprived of such association results in retarded growth and metabolism whilst they loss high plant biomass. This is also in conformity with the findings of Hamilton et al. [18] and Hamilton and Bauerle [31].

\section{ROS generation and oxidative stress modulation}

It was found that the activities of antioxidants and related enzymes were significantly higher in endophyteassociated plants under osmotic imbalance induced ROS generation. With or without osmotic stress, endophyte elicitation has significantly regulated the antioxidant activities as compared to control and sole SA treated plants. It was shown that the responses of ROS generation and antioxidant signaling were similar to the effects caused by pathogenic and mutualistic microorganisms [42]. As both are different forms of consortiums however, higher antioxidant generation can improve plant defenses against disease and abiotic stress conditions. This was further elucidated by White and Torres [42] and Hamilton et al. [18]. Stress oriented ROS generations are minimized by the antioxidant and related enzymes production insides hostcells. ROS accumulation, on the other hand, has deleterious impacts on the functional membrane and macro-organelles. ROS are removed from the cell directly (catalase and peroxidase) or indirectly (redox molecules like glutathione).

The present findings showed higher levels of glutathione and total polyphenol and lower levels of lipid peroxidation and superoxide anion formation in the 
pepper plants associated with $P$. resedanum. The effects were more significant in $\mathrm{SA}+\mathrm{EA}$ treated plants. It indicated that membrane injury was lower in endophyteassociated plants (EA and $\mathrm{SA}+\mathrm{EA}$ ) as the plants had lesser electrolytic leakage and lipid peroxidation (MDA content). Since membrane bounded lipid hydroperoxides are difficult to measure due to their instability, therefore we measured the degree of lipid peroxidation to quantify secondary breakdown products like MDA. Higher ROS, on the other hand, autocatalyze peroxidation of lipid membrane and affect membrane semi-permeability under high drought stress. Activation of antioxidant scavengers can enhance membrane stability against ROS attack while MDA content can be used to assess the stress injury of plants [43].

In stress related antioxidant enzymes, higher catalase (CAT), peroxidase (POD), and polyphenol oxidase (PPO) activities were observed in endophyte-infected plants as compared to non-infected control and sole SAtreated plants. CAT, POD and PPO have also been known to articulate the ROS induced oxidative burst. Increased catalase activity is associated with increased root length and enhanced seedling growth as shown by Harman [40]. Similarly, peroxidase and is polyphenol oxidase protects cells against the destructive influence of $\mathrm{H}_{2} \mathrm{O}_{2}$ by catalyzing its decomposition through the oxidation of phenolic osmolytes [44]. Previously, researchers have identified the crop growth regulation under stress conditions through activation of CAT, POD and PPO $[20,31,45]$. Similarly, the importance of endophyte colonization in terms of antioxidant activity and ROS production has been shown significance and often positive for the host-plant fitness [46], however this could be further verified by further experiments in case of $P$. resedanum.

\section{Co-synergism of SA with endophyte under osmotic stress} The SA application to the pepper plants had a growth promoting effect as compared to control plants. The SA also helped the plants to counteract the negative effects of osmotic stress. The effect of SA and EA on pepper shoot growth, chlorophyll contents was almost similar as compared to SA+EA treatments but this effect was significantly higher than control plants. Exogenous SA is known for its role in abiotic stress mitigation. In recent past, SA application has evidenced improved plant growth against abiotic stress [47-49]. Previous studies have shown that SA application to maize plant helped in alleviating the negative effects on the plants under drought stress [49]. Similarly, in case of tomato and bean plants, SA application has increased the plant biomass and decreased the drought induced oxidative stress by increasing the enzymatic activities [50]. Agarwal et al. [45] and Horvath et al. [9] also observed that SA application can improve plant biomass and enhance the antioxidant response against osmotic stress. The same is shown in our findings when we applied SA to pepper plants as compared to control plants.

During endophytic-fungal association, it was observed that the SA application to EA plants significantly increased the growth and metabolism as compared to sole SA and control plants. Furthermore, the biomass loss was much pronounced in non-inoculated and sole SA plants as compared to EA and SA+EA plants. Previously, it was shown that exogenous SA to roots of fungal-inoculated rice does not inhibit the root colonization of fungi [12]. Ludwig-Müller et al. [13] also reported that exogenous SA did not effected the root colonization by growth promoting fungi. However, our data shows the increased endophytic-colonization in SA treated host plants. This was also conformity to the results of Liu et al. [19], who indicated that exogenous SA application to fungal (Glomus mosseae) inoculated Avena nuda plants has increased the abiotic stress tolerance and had beneficial impacts on fungal colonization. The SA application to endophyte-inoculated plants not only increased endophytes abundance but also increased the host plant biomass, antioxidants and endogenous SA contents. It was shown that endogenous SA increased in endophyte-inoculated plants treated with SA as compared to sole SA and control plants under osmotic stress conditions.

Increased endogenous SA and antioxidant activities play an important role in abiotic and biotic defense signaling $[47,48]$. Under abiotic stress, high endogenous SA may mitigate the negative effects of ROS accumulation. Such functions can counteract the adverse effects of stress under mutualistic relationship as SA initiates induced systemic resistance [51]. Enhanced SA levels are especially important to reduce the susceptibility of plants to biotic and abiotic stresses [51]. We assume that the ISR stimulated through endophyte association activated the SA responses during osmotic stress. Mutualistic relationship initiates ISR and improves plant performance against biotic and abiotic stresses [43]. However, this concept is still overlooked in endophyte-induced ISR. Although Penicillium spp. have been known as potential inducers of ISR in various plants [11], our scientific understanding of the molecular mechanisms by which Penicillium sp. influence the outcome of plant abiotic stress tolerance is still marginal.

\section{Conclusion}

Fungal endophyte, $P$. resedanum not only improves plant growth but also extend greater benefits to the host-plants to mitigate the negative effects of gradual osmotic stress. Exogenous SA application to pepper plant improved the stress tolerance of the plants while in 
combination with endophyte-inoculation it further regulated the stress impacts. The oxidative stress and cellular injury were reduced in the presence of endophyte and SA application. A significantly higher endogenous SA accumulation during endophytic fungal interaction and stress could be attributed to extend the tolerance against stress.

\section{Additional file}

Additional file 1: Table S1. HPLC conditions used for salicylic acid analysis.

\section{Authors' contributions}

ALK planned and undertaken the research project. ALK performed the experiments, analyzed the data and drafted the manuscript. $\mathrm{MH}, \mathrm{MW}$ and IJL had undertaken the plant hormonal work. AA and AA helped in revision of the MS and statistical analysis. All Authors contributed in writing the manuscript and approved its final content.

\section{Acknowledgements}

The research work was supported by Eco-Innovation Project, Korean Government's R \& D program on Environmental Technology and Development. The authors are also thankful to Prof. Hee-Young Jung, Kyunpook National University, South Korea for his help in microscopic analysis.

\section{Author details}

'Department of Biological Sciences and Chemistry, University of Nizwa, Nizwa, Oman. ${ }^{2}$ School of Applied Biosciences, Kyungpook National University, Daegu, Republic of Korea. ${ }^{3}$ Kohat University of Science \& Technology, Kohat, Pakistan. ${ }^{4}$ Department of Botany, Abdul Wali Khan University, Mardan, Pakistan.

Received: 9 December 2012 Accepted: 20 February 2013

Published: 1 March 2013

\section{References}

1. Hirayama T, Shinozaki K: Research on plant abiotic stress responses in the post-genome era: past, present and future. Plant J 2010, 61(6):1041-1052.

2. Jakab R, Ton J, Flors V, Zimmerli L, JP Mt, Mauch-Mani B: Enhancing Arabidopsis Salt and Drought Stress Tolerance by Chemical Priming for Its Abscisic Acid. Plant Physiol 2005, 139:267-274.

3. Im YJ, Ji M, Lee A, Killens R, Grunden AM, Boss WF: Expression of Pyrococcus furiosus superoxide reductase in Arabidopsis enhances heat tolerance. Plant Physiol 2009, 151:893-904

4. Hayat $Q$, Hayat $S$, Irfan $M$, Ahmad A: Effect of exogenous salicylic acid under changing environment: A review. Environ Exp Botany 2010, 68:14-25.

5. Saruhan N, Saglam A, Kadioglu A: Salicylic acid pre-treatment induces drought tolerance and delays leaf rolling by inducing antioxidant systems in maize genotypes. Acta Physiol Plant 2012, 34:97-106.

6. Dat JF, Foyer $\mathrm{CH}$, Scott IM: Changes in salicylic acid and antioxidants during induced thermotollerance in mustard seedlings. Plant Physiol 1998, 118:1455-1461

7. Farooq M, Aziz T, Basra SMA, Cheema MA, Rehman H: Chilling tolerance in hybrid maize induced by seed priming with salicylic acid. J Agron Crop Sci 2008, 194:161-168.

8. Sakhabutdinova AR, Fatkhutdinova DR, Bezrukova MV, Shakirova FM: Salicylic acid prevents the damaging action of stress factors on wheat plants. In Proceedings of the European Workshop on Environmental Stress and Sustainable Agriculture; 7-12 September 2003. Edited by V. Alexieva. Oulu, Finland: Academy Publisher Inc:; 2003:314-319.

9. Horvath E, Pal M, Szalai G, Paldi E, Janda T: Exogenous 4-hydroxybenzoic acid and salicylic acid modulate the effect of short-term drought and freezing stress on wheat plants. Biol Plantarum 2007, 51:480-487.
10. Hussain M, Malik MA, Faroog M, Ashraf MY, Cheema MA: Improving drought tolerance by exogenous application of glycinebetaine and salicylic acid in sunflower. J Agron Crop Sci 2008, 194:193-199.

11. Herrera-Medina MJ, Gagnon H, Piche Y, Ocampo JA, Garrido JMG, Vierheilig $\mathrm{H}$ : Root colonization by arbuscular mycorrhizal fungi is affected by the salicylic acid content of the plant. Plant Sci 2003, 164:993-998.

12. Hossain M, Sultana F, Kubota M, Koyama H, Hyakumachi M: The Plant Growth-Promoting Fungus Penicillium simplicissimum GP17-2 Induces Resistance in Arabidopsis thaliana by Activation of Multiple Defense Signals. Plant Cell Physiol 2007, 48:1724-1736.

13. Ludwig-Müller J, Bennett RN, García-Garrido JM, Piché Y, Vierheilig H: Reduced arbuscular mycorrhizal root colonization in Tropaeolum majus and Carica papaya after jasmonic acid application cannot be attributed to increased glucosinolate levels. J Plant Physio/ 2002, 159:517-523.

14. Rodriguez RJ, Elizabeth $J H$, Marshal V, Leesa H, Beckwith LB, Kim Y, Redman RS: Stress tolerance in plants via habitatadapted symbiosis. ISME J 2008, 2:404-416

15. Waller F, Achatz B, Baltruschat H, Fodor J, Becker K, Fischer M, Heier T, Huckelhoven R, Neumann C, Von-Wettstein D, Franken P, Kogel KH: The endophytic fungus Piriformis indica reprograms barley to saltstress tolerance, disease resistance and higher yield. PNAS 2005, 102:13386-13391.

16. Redman RS, Kim YO, Woodward CJDA, Greer C, Espino L, Doty SL, Rodriguez RJ: Increased fitness of rice plants to abiotic stress via habitat adapted symbiosis: a strategy for mitigating impacts of climate change. PLoS One 2011, 6:e14823. doi:10.1371/journal.pone.0014823.

17. Khan AL, Hamayun M, Kim YH, Kang SM, Lee IJ: Ameliorative symbiosis of endophyte (Penicillium funiculosum LHL06) under salt stress elevated plant growth of Glycine max L. Plant Physiol Biochem, 49:852-862.

18. Hamilton CE, Dowling TE, Faeth SH: Hybridization in Endophyte Symbionts alters host response to moisture and nutrient treatments. Microb Ecol 2010, 59:768-775.

19. Li R, Jiang Y, Xu J, Zhou B, Ma C, Liu C, Yang C, Xiao Y, Xu Q, Hao L: Synergistic Action of Exogenous Salicylic Acid and Arbuscular Mycorrhizal Fungus Colonization in Avena nuda Seedlings in Response to $\mathrm{NO}_{2}$ Exposure. Bull Environ Cont Toxicol 2010, 84:96-100.

20. Liu HP, Dong BH, Zhang YY, Liu ZP, Liu YL: Relationship between osmotic stress and the levels of free, conjugated and bound polyamines in leaves of wheat seedlings. Plant Sci 2004, 166:1261-1267.

21. Kumar DSS, Hyde KD: Biodiversity and tissue-recurrence of endophytic fungi in Tripterygium wilfordii. Fungal Diversity 2004, 17:69-90.

22. Ellman GL: Tissue sulfhydryl groups. Archives Biochem Biophys 1959, 82:70-77.

23. Kumazawa S, Hamasaka T, Nakayama T: Antioxidant activity of propolis of various geographic origins. Food Chem 2004, 84:329-339.

24. Doke N: Involvement of superoxide anion generation in the hypersensitive response of potato tuber tissues to infection with an incompatible race of Phytophthora infestans and to the hyphal wall components. Physiol Plant Path 1983, 23:345-357.

25. Ohkawa H, Ohishi N, Yagi K: Assay of lipid peroxides in animal tissue by thiobarbituric acid reaction. Anal Biochem 1979, 95:351-358.

26. Bradford MM: A rapid and sensitive method for the estimation of microgram quantities of protein utilizing the principle of protein-dye binding. Anal Biochem 1976, 72:248-254.

27. Aebi H: Catalase in vitro. Methods Enzymol 1984, 105:121-127.

28. Kar M, Mishra D: Catalase, peroxidase, and polyphenoloxidase activites during rice leaf senescence. Plant Physiol 1976, 57:315-319.

29. Seskar M, Shulaev V, Raskin I: Endogenous methyl salicylate in pathogeninoculated tobacco plants. Plant Physiol 1998, 116:387-392.

30. Rodriguez $\mathrm{R}$, Redman $\mathrm{R}$ : More than $\mathbf{4 0 0}$ million years of evolution and some plants still can't make it on their own: plant stress tolerance via fungal symbiosis. J Exp Bot 2008, 59:1109-1114.

31. Hamilton CE, Bauerle TL: A new currency for mutualism? Fungal endophytes alter antioxidant activity in hosts responding to drought. Fungal Div 2012, 54:39-49.

32. Schulz B, Boyle C: The endophytic continuum. Myco Res 2005, 109:661-686.

33. Singh LP, Gill SS, Tuteja N: Unraveling the role of fungal symbionts in plant abiotic stress tolerance. Plant Signal Behav 2011, 6:175-191.

34. Firáková S, Šturdíková M, Múčková M: Bioactive secondary metabolites produced by microorganisms associated with plants. Biologia 2007, 62:251-257. 
35. Hamayun M, Khan SA, Khan AL, Rehman G, Kim YH, Iqbal I, Hussain J, Sohn EY, Lee IJ: Gibberellin production and plant growth promotion from pure cultures of Cladosporium sp. MH-6 isolated from Cucumber (Cucumis sativus. L). Mycologia 2010, 102:989-995.

36. Kowaide H: Molecular and biochemical analysis of Gibberellins biosynthesis in Fungi. Biosci Biotechnol Biochem 2006, 70:583-590.

37. Bömke C, Rojas MC, Gong F, Hedden P, Tudzynski B: Isolation and characterization of the gibberellin biosynthetic gene cluster in Sphaceloma manihoticola. Appl Environ Microbiol 2008, 74:5325-5339.

38. Khan SA, Hamayun M, Yoon HK, Kim HY, Suh SJ, Hwang SK, Kim JM, Lee IJ, Choo YS, Yoon UH, Kong WS, Lee BM, Kim JG: Plant growth promotion and Penicillium citrinum. BMC Microbiol 2008, 8:231-239.

39. Young CA, McMillan L, Telfer E, Scott B: Molecular cloning and genetic analysis of an indole-diterpene gene cluster from Penicillium paxilli. Mol Microbiol 2001, 39:754-764.

40. Harman GE: Multifunctional fungal plant symbionts: new tools to enhance plant growth and productivity. New Phytol 2011, 189:647-649.

41. Foyer $\mathrm{CH}$, Shigeoka S: Understanding oxidative stress and antioxidant functions to enhance photosynthesis. Plant Physiol 2011, 155:93-100

42. White JF, Torres MS: Is plant endophyte-mediated defensive mutualism the result of oxidative stress protection? Physiol Plant 2010, 138:440-446

43. Elwan MWM, El-Hamahmy MAM: Improved productivity and quality associated with salicylic acid application in greenhouse pepper. Scientia Horticul 2009, 122:521-526.

44. Elmi A, West C: Endophyte infection effects on stomatal conductance, osmotic adjustment and drought recovery of tall fescue. New Phytol 1995, 131:61-67.

45. Agarwal S, Sairam RK, Srivastava GC, Meena RC: Changes in antioxidant enzymes activity and oxidative stress by abscisic acid and salicylic acid in wheat genotypes. Biologia Plantarum 2005, 49(4):541-550.

46. Mittler R, Vanderauwera S, Gollery M, Breusegem FV: Reactive oxygen gene network of plants. Trends Plant Sci 2004, 9:1360-1385.

47. Lee S, Kim SG, Park CM: Salicylic acid promotes seed germination under high salinity by modulating antioxidant activity in Arabidopsis. New Phytol 2010, 188:626-637.

48. Yuan S, Lin HH: Role of salicylic acid in plant abiotic stress. Zeitschrift für Naturforschung 2008, 63:313-320.

49. Janda K, Hideg E, Szalai G, Kovács L, Janda T: Salicylic acid may indirectly influence the photosynthetic electron transport. J Plant Physiol 2012 doi:10.1016/j.jplph.2012.02.020.

50. Singh B, Usha K: Salicylic acid induced physiological and biochemical changes in wheat seedlings under water stress. Plant Growth Regul 2003, 39:137-141.

51. Alonso-Ramirez A, Rodriguez D, Reyes D, Jimenez JA, Nicolas G, LopezCliment M: Evidence for a role of gibberellins in salicylic acid-modulated early plant responses to abiotic stress in Arabidopsis seeds. Plant Physiol 2009, 150:1335-1344.

doi:10.1186/1471-2180-13-51

Cite this article as: Khan et al.: Co-synergism of endophyte Penicillium resedanum LK6 with salicylic acid helped Capsicum annuum in biomass recovery and osmotic stress mitigation. BMC Microbiology 2013 13:51.

\section{Submit your next manuscript to BioMed Central and take full advantage of:}

- Convenient online submission

- Thorough peer review

- No space constraints or color figure charges

- Immediate publication on acceptance

- Inclusion in PubMed, CAS, Scopus and Google Scholar

- Research which is freely available for redistribution 